\title{
Age-related Normal Tolerance Intervals for Retinal Vascular Response to Systemic Autonomic Nerve Stimulation
}

\author{
D. W. HILL, L. P. LANIGAN, C. V. CLARK \\ London
}

\begin{abstract}
Summary
This study establishes the age-adjusted tolerance intervals for retinal vascular responses to sustained handgrip contraction (a test of sympathetic nerve function). Forty adult subjects (mean age: $39.7 \pm 14.1$ years, range: 20 to 72 years; 19 males) were included in the final analysis. Mean percentage calibre change in response to sustained handgrip contraction in this population was $-\mathbf{5 . 6 1} \%$ with a significant correlation between subject age and percentage retinal vessel calibre change $(\mathbf{p}<\mathbf{0 . 0 0 1})$. Mean retinal arteriolar calibre change in response to systemic autonomic stimulation varied significantly with age: between $-8.48 \%$ at age 20 years and $-2.06 \%$ at age 70 years with a mean of $-5.95 \%$ at the mean age of 39.7 years. The standard deviation is $3.04 \%$ and the $95 \%$ confidence intervals were set between $\pm 6.17 \%$. This autoregulatory reflex appears to be inversely related to age.
\end{abstract}

Recent studies have demonstrated significant constriction of the retinal vasculature in response to systemic stimulation of the autonomic nervous system. ${ }^{1,2}$ Sustained isometric muscle contraction (by handgrip dynamometry) provokes a generalised discharge of sympathetic nerves, and the resultant elevation in diastolic blood pressure is an established indication of sympathetic nerve function. ${ }^{3}$ Although the rise in blood pressure and retinal vascular constriction were contemporaneous, there was no correlation between them, and therefore the vascular reflexes are unlikely to represent a purely retinal myogenic response to increased blood pressure. It has been proposed that the observed autoregulatory response of the retinal microcirculation is effected in part, by the autonomic nervous system, a hypothesis supported by evidence of impaired retinal vascular reflexes in subjects with autonomic neuropathy $\mathrm{y}^{2}$ or transection of the cervical sympathetic chain. ${ }^{4}$
The aim of the present study was to define the age-adjusted tolerance intervals for retinal vascular responses to systemic autonomic nerve stimulation in normal subjects, thereby establishing the variability of the normal response.

\section{Patients and Methods}

Fifty healthy adult patients were included in the study. The patients were randomly selected from the general clinics and casualty department at Moorfields Eye Hospital, where they were attending for minor ophthalmic complaints. No subject had any ophthalmic disease at the time of study and patients with a past history of significant ocular disease were excluded from assessment. Subjects taking routine prescribed medication-ocular or systemic - were similarly excluded from the study. Informed consent was obtained from all participants. Retinal vascular responses to systemic autonomic nerve stimu- 
lation were determined by the following technique.

Each subject was studied seated at a fundus camera with one pupil dilated (selected by the subject) with g. tropicamide $1 \%$. All photographs were taken by the same observer using a wide angle Cannon fundus camera (CF 60-S) which was triggered during the diastolic phase of the pulse cycle, using a finger-tip pulse detector coupled to an adjustable time delay. Five photographs were taken in quick succession at approximately one second intervals at each phase.

Baseline photographs were taken followed by three baseline measurements of blood pressure recorded from the non-exercising arm using an automatic sphygmomanometer (COPAL-UA 231). The subject then performed three maximum handgrip contractions, each lasting less than two seconds, using the dominant arm to establish the maximum voluntary contraction (MVC). A laboratory built strain gauge handgrip dynamometer with continuous chart recording was used; it had an adjustable audible alarm set at $33 \%$ of each subjects MVC for 2.5 minutes. During this exercise period lasting 2.5 minutes, three phases of fundus photographs were taken at $0.5,1.5$ and 2.5 minutes. On recovery one phase of fundus photographs and a final blood pressure reading were taken five minutes after release of sustained handgrip contraction.

All photographs were recorded on Ilford FP4 film with a red-free filter and developed in PQ Universal $(1+19)$ for 12 minutes at $20^{\circ} \mathrm{C}$ with sensitometric control. ${ }^{5}$

\section{Calibre measurement}

The films were analysed using the Quantimet 800 Image analyser (Cambridge Instruments). ${ }^{5}$ Nine to 18 suitable sites were measured in each subject comprising similar numbers of arterioles and veins: only the arteriolar measurements were considered in this paper. A typical fundus photograph illustrating sites of measurement in one subject is shown in Fig 1. All calibre measurements of these vessel sites were stored on floppy disc for later retrieval and statistical analysis. Means and standard deviations for each set of replications in each phase were calculated. A total of 373 arteriolar sites were measured.
From the mean values for each site for each phase, the percentage calibre change in response to handgrip was calculated (percentage change $=\frac{\bar{X}_{c}-\bar{X}_{c} \times 100}{\bar{X}_{c}}$ where symbol $\bar{X}_{c}$ is the experimental and $\bar{X}_{c}$ the control mean). The experimental mean was taken from that phase during the handgrip where the mean change of all arterioles was at a maximum, or if two phases had a similar mean, then the phase with the more uniform response was selected, as judged by the standard deviation of the mean.

The results showed a wide variation in the accuracy of measurements, and a trend for large values of constrictor response to occur where the accuracy of measurement was reduced, as evidenced by higher values of the original replication $\mathrm{SD}$ in either phase. To avoid the introduction of bias in the calculation of tolerance intervals, an empirical cut off point was established by inspection of the data, and those sites which had an original phase replication $\mathrm{SD}>17 \times 10^{-3}$ in either control or experimental phase, or where the common SD for both phases was $>13 \times 10^{-3}$ were excluded. We shall refer to this as the '17/13 rule'.

\section{Results}

Fifty subjects were studied, but the photographs of four were of poor quality, leaving 46 to be included in the initial analysis, 22 male and 24 female. The age range was $20-74$ years and the mean 46.6 years $\pm 15.04(\mathrm{SD})$. A total

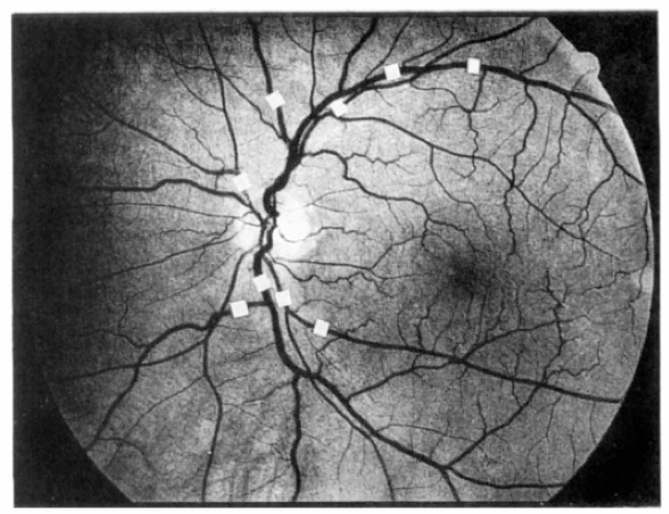

Fig. 1. Fundus photograph showing typical sites of measurement. 
Table I Mean percent calibre change = top; no of sites $=$ bottom. The top right and bottom compartments cover large $S D$ ranges. The bottom right compartments contain too little data to be of value.

\begin{tabular}{|c|c|c|c|c|c|}
\hline \multirow{9}{*}{$\begin{array}{l}\text { Control } \\
\mathrm{SD} \times 10^{-3}\end{array}$} & \multicolumn{5}{|c|}{ Experimental $S D \times 10^{-3}$} \\
\hline & & $1-8$ & $9-16$ & $17-24$ & $25+$ \\
\hline & \multirow{2}{*}{$1-8$} & -5.54 & -5.63 & -7.08 & \multirow[t]{3}{*}{-11.44} \\
\hline & & 132 & 54 & 12 & \\
\hline & \multirow{2}{*}{$9-16$} & -6.88 & -8.06 & -11.5 & \\
\hline & & 40 & 32 & 10 & 41 \\
\hline & \multirow{2}{*}{$17-24$} & -11.18 & -12.10 & & \\
\hline & & 11 & 10 & & \\
\hline & $25+$ & -10.13 & 15 & & \\
\hline
\end{tabular}

of 373 arteriolar sites were measured; the mean change in vessel calibre in response to handgrip was $-7.26 \pm 8.29 \%$. Table I demonstrates the trend towards increasing constriction in the groups with higher phase replication SD, and Table II shows a more detailed analysis of the constriction response in relation to phase replication SD.

Imposition of the arbitrary ' $17 / 13$ ' exclusion rule to individual sites reduced the population analysed to 235 sites, and excluded one male subject. Figure 2 shows the age and sex distribution of the 45 subjects included in the final analysis; the median age for males $(n=21)$ was 35 years, range: 21-74 years; and for females 44 years, range: $20-72$ years. There was no significant difference in retinal arteriolar responses between the sexes. When these

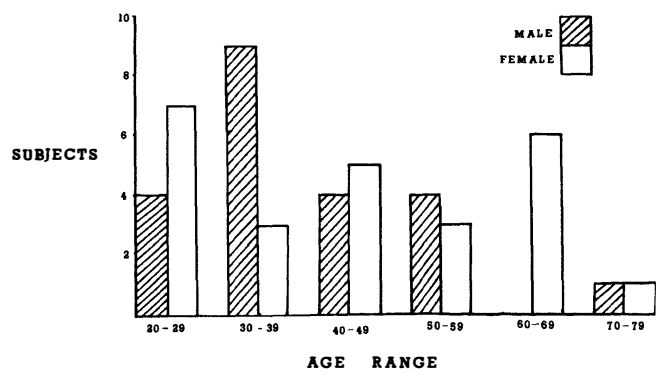

Fig. 2. Age and sex distribution of subjects included in the analysis under the '17/13 rule'. subjects with six or more arteriolar sites admissible for analysis ('17/13' rule) were examined, the mean retinal vessel calibre change for men $(n=8)$ was $-6.94 \pm 4.22 \%$ and $-5.18 \pm 3.58 \%$ for women $(n=11)$, $\mathrm{t}_{17}=0.98 ; 0.5>\mathrm{p}>>0.2$.

There was no convincing evidence that there was any correlation between initial vessel diameter and its percentage calibre change. When the data was subclassified according to age, only one decade (30-39 years) showed a significant correlation between initial vessel diameter and the degree of vascular constriction, $0.02>\mathrm{p}>0.01$.

The number of sites measured for each subject varied from one to 11 with a median value of five; Figure 3 shows the frequency distribution. The mean retinal arteriolar calibre change in response to handgrip was $-5.62 \pm 5.15 \%$ for the 235 sites. The initial calibres recorded on film varied from 0.111 to $0.264 \mathrm{~mm}$ with a mean value of $0.156 \pm 0.024 \mathrm{~mm}$ (nominally equivalent to 0.58 microns at the fundus as calculated using the Gullstrand schematic eye).

Table II fragments the data to enable the elimination of those sites at which the large replication variance is associated with an apparently increasing constriction in the experimental phase: when the mean percentage calibre change for each of the remaining 
Table II Mean per cent calibre change = top; $S D=$ centre; number of sites $=$ bottom in each compartment. All compartments above and left of the double line have control and experimental $S D<17$ and common $S D<13$.

Control

$\mathrm{SD} \times 10^{-3}$

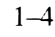

Experimental $S D \times 10^{-3}$

$5-8$

$9-12$

$1-4$

$5-8$

$9-12$

$13-16$

\begin{tabular}{|c|c|c|c|}
\hline-5.93 & -5.52 & -6.55 & -6.33 \\
\pm 4.61 & \pm 5.66 & \pm 4.39 & \pm 6.39 \\
28 & 31 & 11 & 15 \\
\hline-4.83 & -6.00 & -5.10 & -5.17 \\
\pm 4.94 & \pm 4.97 & \pm 6.07 & \pm 4.40 \\
42 & 31 & 21 & 6 \\
\hline-7.45 & -6.53 & -6.90 & -8.00 \\
\pm 5.85 & \pm 5.21 & \pm 7.91 & \pm 6.70 \\
11 & 17 & 10 & 15 \\
\hline-2.67 & -8.56 & -13.73 & -8.33 \\
\pm 6.11 & \pm 4.90 & \pm 7.93 & \pm 12.66 \\
3 & 9 & 4 & 3 \\
\hline \hline
\end{tabular}

13 cells, within the ' $17 / 13$ rule' is examined against the probability that it will occur in a sample of appropriate size drawn from the whole population of 235 sites, it is seen that seven samples fall within the $p=0.5$ boundary, five within $0.5>\mathrm{p}>0.2$ and only one within $0.2>\mathrm{p}>0.1$ (Fig 4 ); evidence that the empirical exclusion process has resulted in a single, normally distributed population.

For the purpose of establishing confidence interval data, the original population of 46 subjects was re-examined and a selected population was assembled, comprising 40

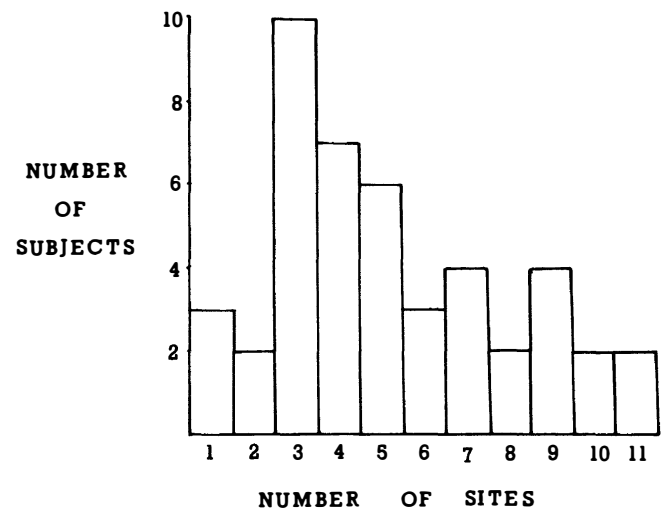

Fig. 3. Frequency distribution of number of sites measured per subject. subjects each of whom had at least three arterial sites conforming to the $17 / 13$ rule and including only such sites. The remaining analyses will be solely concerned with this selected population, which has the following parameters: $n=40$, mean age $39.7 \pm 14.1$ years, range $20-72$ years; 19 males (mean age: $38.1 \pm 10.2$ years, range $21-59$ years) and 21 females (mean age: $41.1 \pm 17.2$ years range 20 to 72 years).

Figure 5 shows a histogram of the frequency distribution of percentage calibre change for this population, with a superimposed normal distribution curve to the same parameters. A

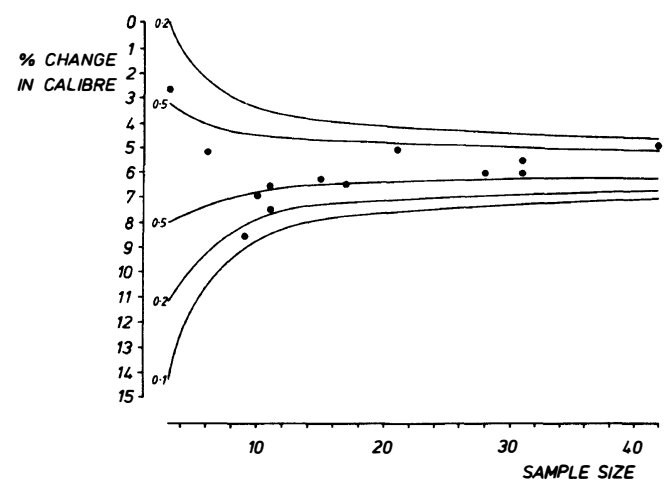

Fig. 4. Adopting the '17/13 rule' results in a normally distributed population. 


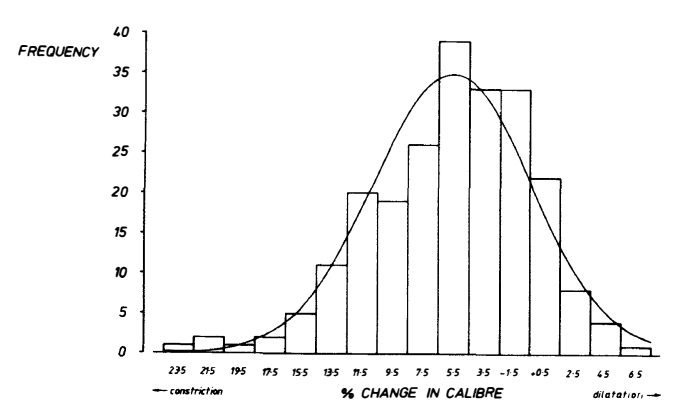

Fig. 5. Percentage calibre change for the population conforming to the '17/13 rule' with a superimposed normal distribution curve.

chi-squared test of the differences between the observed and expected values yields values of 13.67 with 14 degrees of freedom, $0.05>\mathrm{p}>0.10$ for the whole distribution and 7.27 with 12 degrees of freedom $0.90>p>0.50$, if the two tail values in the extreme left end of the distribution are omitted.

Although the grand mean percentage calibre change in response to sustained handgrip contraction in this population is $-5,61 \%$ there is a significant correlation between subject age and percentage calibre change: $\mathrm{y}($ calibre change $)=-11.04+0.13 \times($ age $)$, $\mathrm{r}=0.52, \mathrm{p}<0.001$. The age-adjusted $95 \%$ tolerance interval for retinal arteriolar constriction to systemic autonomic stimulation is shown in Figure 6. There is also a significant correlation between the initial diastolic blood pressure and the percentage vascular calibre

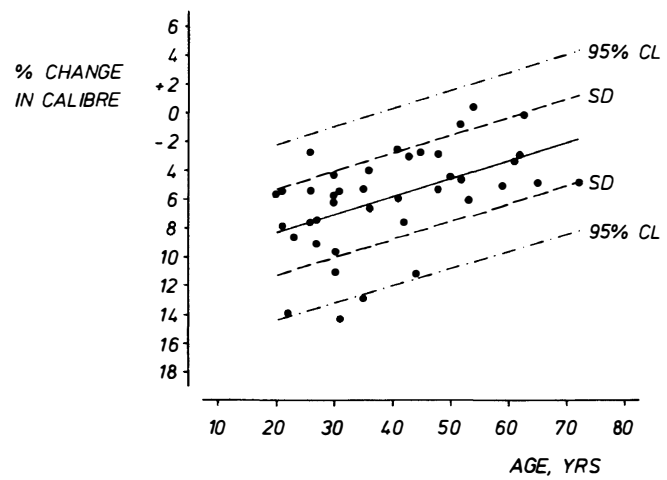

Fig. 6. Age-adjusted $95 \%$ tolerance intervals for retinal arteriolar responses to systemic autonomic stimulation. change: $y$ (calibre change $)=-20.71+0.18$ $\times($ initial diastolic blood pressure $)$, $\mathrm{r}=+0.51 ; \mathrm{p}<0.001$ (Fig 7).

These two factors age and initial diastolic blood pressure are not independent variables and indeed a correlation exists between them: $\mathrm{y}$ (initial diastolic blood pressure) $=$ $68.03+0.32 \times($ age $), r=0.46, p<0.005$, (Fig $8)$.

Figure 6 clearly demonstrates that mean retinal arteriolar calibre change in response to systemic autonomic stimulation varies significantly with age, between $-8.48 \%$ at age 20 years and $-2.06 \%$ at age 70 years with a mean of $-5.95 \%$ at the mean age (39.7 years). The standard deviation is $3.04 \%$ and the $95 \%$ confidence intervals are set between $\pm 6.17 \%$.

There is no correlation between elevation of diastolic blood pressure and retinal arteriolar constriction in response to sustained handgrip contraction: $r=-0.09, \quad y \quad$ (calibre change $)=-4.72-0.04 \times($ diastolic blood pressure), $\mathrm{p}>0.10$. There is also no evidence of a significant difference in the response of male and female subjects, nor does the initial calibre appear to influence the percentage change within the limits of the study.

\section{Discussion}

Acute onset vasoconstriction of the retinal microcirculation has been demonstrated in response to systemic stimulation of the autonomic nervous system. ${ }^{1}$ This reponse is statistically reproducible and provides an effective method whereby autoregulation of the retinal vasculature may be manipulated by a safe, non-invasive, clinically applicable technique. Impariment of retinal vascular autoregulation has subsequently been demonstrated in diabetic patients with autonomic neuropathy ${ }^{2}$ and in subjects with traumatic section of sym-

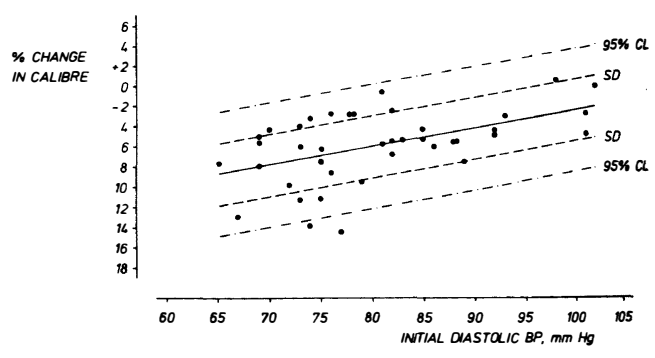

Fig. 7. Correlation between initial diastolic blood pressure and percentage vascular calibre change. 


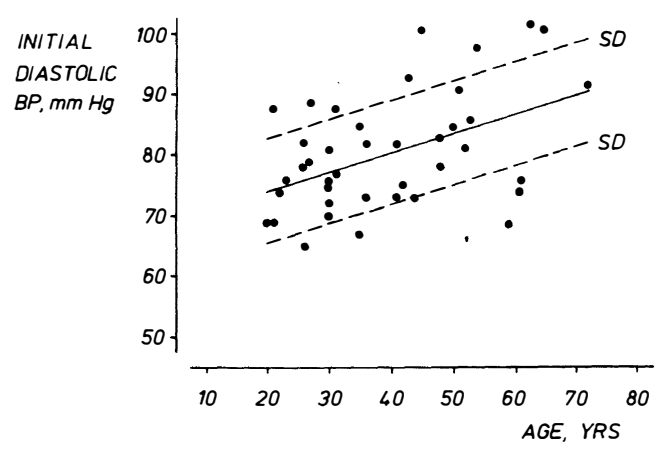

Fig. 8. Correlation between age and initial diastolic blood pressure.

pathetic denervation to the eye, ${ }^{4}$ in both instances elevation of diastolic blood pressure produced by handgrip resulted in reduced retinal vascular response thus supporting the hypothesis that the autonomic nervous system affects the regulation of retinal blood flow. The present study has established ageadjusted normal tolerance intervals for this reflex, demonstrating a significant correlation between the retinal vascular response and age.

The analytical treatment of the data collected has been complex because of the variable accuracy of measurement, due to errors accumulating in the multistage process, fundus photography, photographic processing and image analysis; no other current method however, allows the instantaneous collection of data for multiple measurement. The criteria applied have allowed the assembly of a homogenous population of data, likely to represent a true random sample, for regression analysis.

The present study has confirmed the presence of a direct correlation between retinal vascular reactivity and age, establishing the $95 \%$ tolerance interval for subjects aged 20 to 74 years. The lower limit of the $95 \%$ tolerance interval for individual site responses intersects zero at age 38 years, thus limiting the usefulness of the test for individual older subjects, though accuracy is improved in proportion to the square root of the number of sites examined; similar groups of subjects can afford useful comparisons in the older age groups. However, as the commonest cause of autonomic neuropathy in the United King- dom is diabetes mellitus, ${ }^{6}$ particularly insulindependent diabetes mellitus which usually has an early age of onset, the tolerance intervals for retinal vascular reactivity are applicable to a significant proportion of these patients in whom one may require specific information on the integrity of the retinal vascular autoregulation. A recent study has demonstrated significant impairment of retinal vascular autoregulation in diabetic patients, particularly those with autonomic neuropathy compared to age-matched normal subjects. ${ }^{2}$

The breadth of these confidence limits may be ascribed to several factors:

(1) The stimulus to retinal vaso-constriction in the handgrip test cannot be accurately quantified, and may vary friom subject to subject, especially as the prescribed level of isometric muscle contraction depends upon a subjective response by the subject who is required to perform a maximal grip as the standard by which the required level is set.

(2) Individual arteriolar sites may vary in their responsiveness; this has been statistically proven in the case of some, but not all subjects. $^{7}$

(3) The inherent variance of the multistage measurement process.

The results of this study have confirmed the presence of vasconstrictive responses of the retinal vasculature to systemic autonomic nerve stimulation in a large series of subjects, a response which is not correlated with the simultaneous rise in blood pressure ${ }^{1}$, and have established age-adjusted normal tolerance intervals for the autoregulatory reflex. An inevitable conclusion is that the autonomous nervous system may exert a significant influence on the autoregulation of retinal blood flow, a reflex which appears to be inversely related to age.

We thank the patients attending Moorfields Eye Hospital, who took part in this study. The project was supported by a grant from the Royal National Institute for the Blind. L. P. Lanigan held a Smith \& Nephew Fellowship award.

Key words: Autonomic nerve function tests, systemic autonomic nerve stimulation, retinal autoregulation, retinal vessel calibres. 


\section{References}

${ }^{1}$ Lanigan LP, Clark CV, Hill DW: Retinal circulation responses to systemic autonomic stimulation. Eye 1988, 2, 412-17.

${ }^{2}$ Lanigan LP, Clark CV, Allawi J, Hill DW, Keen H: Responses of the retinal circulation to systemic autonomic nerve stimulation in diabetes mellitus. Eye 1989, 3: 39-47.

${ }^{3}$ Ewing DJ, Irving JB, Kerr F, Wildsmith JAW, Clarke $\mathrm{BF}$ : Cardiovascular responses to sustained handgrip in normal subjects and in patients with diabetes mellitus-a test of autonomic nerve function. Clin Sci Mol Med 1974, 46: 295-306.
${ }^{+}$Lanigan LP, Birche R, Clark CV, Hill DW: The effect of cervical sympathectomy on retinal vessel responses to systemic autonomic stimulation. Eye 1990, 4: 181-9.

${ }^{5}$ Hill DW and Crabtree A: Vascular calibres. Trans Ophthalmol Soc UK 1984, 104: 107.

${ }^{6}$ Watkins BJ and Edmonds ME: Clinical presentation of diabetic autonomic failure. In: Bannister ed. Autonomic Failure, Oxford, Oxford Univ Press 1983:332.

${ }^{7}$ Hill DW: Variability of retinal vessel responses. Folia Ophthalmol 1990,15:275-81. 